\title{
Impaired Mesopic Visual Acuity in Eyes with Early Age- Related Macular Degeneration
}

\author{
María C. Puell, ${ }^{1}$ Ana R. Barrio, ${ }^{1}$ Catalina Palomo-Alvarez,${ }^{1}$ Fernando J. Gómez-Sanz, ${ }^{2}$ \\ Amaya Clement-Corral, ${ }^{2}$ and María J. Pérez-Carrasco ${ }^{1}$
}

Purpose. To determine photopic and mesopic distance highcontrast visual acuity (HC-VA) and low-contrast visual acuity (LC-VA) in eyes with early age-related macular degeneration (AMD).

Methods. Measurements were made in 22 subjects with early AMD and 28 healthy control subjects. Inclusion criteria included a photopic HC-VA of $20 / 25$ or better. Distance VA was measured using HC (96\%) and LC (10\%) Bailey-Lovie logMAR letter charts under photopic $\left(85 \mathrm{~cd} / \mathrm{m}^{2}\right)$ and mesopic $\left(0.1-0.2 \mathrm{~cd} / \mathrm{m}^{2}\right)$ luminance conditions.

Results. Mean mesopic distance HC-VA and LC-VA were significantly worse $(0.1 \log$ MAR and $0.28 \log$ MAR, respectively) in the early AMD group than in the control group. Under mesopic conditions, the mean difference between LC-VA and HC-VA was significantly greater in the early AMD $(0.45$ logMAR) than the control group ( 0.27 logMAR). Mean differences between mesopic versus photopic HC-VA and mesopic versus photopic LC-VA were significantly greater in the early AMD than the control group (0.13 and 0.32 logMAR of difference between the means, respectively). Sensitivity and specificity were significantly greater for mesopic LC-VA than for mesopic HC-VA (Receiver Operating Characteristics, area under the curve [AUC], $0.94 \pm 0.030$ and $0.76 \pm 0.067$, respectively). AUC values for photopic HC-VA and LC-VA were below 0.70 .

Conclusions. Visual acuity testing under low luminance conditions emerged as an optimal quantitative measure of retinal function in early AMD. (Invest Ophthalmol Vis Sci. 2012;53:7310-7314) DOI:10.1167/iovs.11-8649

ge-related macular degeneration (AMD) is the leading cause
many irreversible vision loss among older adults. In 2000, as
threatening stages of AMD (choroidal neovascularization
[CNV] and geographic atrophy [GA]), and many more had
asymptomatic early-stage disease. ${ }^{1}$ According to the Interna-

From the ${ }^{1}$ Applied Vision Research Group, Complutense University, Madrid, Spain; and the ${ }^{2}$ Hospital del Henares, Madrid, Spain.

Supported by Fundación Investigación Médica Mutua Madrileña Grant FMM-02538/2008 and Banco Santander-Universidad Complutense de Madrid Grant GR 58/08.

Submitted for publication September 23, 2011; revised March 28, July 6, and August 21, 2012; accepted September 25, 2012.

Disclosure: M.C. Puell, None; A.R. Barrio, None; C. PalomoAlvarez, None; F.J. Gómez-Sanz, None; A. Clement-Corral, None; M.J. Pérez-Carrasco, None

Corresponding author: María C. Puell, School of Optics and Optometry, Complutense University, Avenida Arcos de Jalón 118, Madrid 28037, Spain; puellma@fis.ucm.es. tional Classification and Grading System for $\mathrm{AMD},{ }^{2}$ the early stage of AMD is characterized by soft drusen and changes in retinal pigment epithelium (RPE) pigmentation associated with soft drusen in the macular region.

Patients with AMD often complain of a worsened quality of vision, especially difficulty in performing activities such as reading and driving at night or in conditions of low illumination. ${ }^{3-5}$ This loss of vision quality occurs early in the course of AMD before any reduction in high-contrast visual acuity (HC-VA) can be detected and before morphological changes in the fundus become clinically apparent. ${ }^{5,6}$ In contrast, it has been possible to correlate the more advanced AMD stages GA and CNV with a loss in VA. ${ }^{7}$

It is known that mesopic vision testing can be sensitive to early signs of retinal disease. ${ }^{8}$ Mesopic luminance, or dim lighting, conditions span approximately three to four log units $\left(0.001-10 \mathrm{~cd} / \mathrm{m}^{2}\right)$ in natural viewing environments. Under such conditions, the rod and cone photoreceptors of the human retina simultaneously convey visual information. Rod dysfunction or selective rod loss has been demonstrated histologically, psychophysically, and electrophysiologically in early AMD and aging. ${ }^{9-12}$ Also, it has been recently reported that rod- and cone-mediated mesopic visual function is significantly reduced in healthy persons with AMD risk genotypes. ${ }^{13}$

Studies addressing visual function in AMD have been extensively reviewed, ${ }^{6,14,15}$ and psychophysical tests of vision, which depend on the functional state of the photoreceptors, have been proposed as a strategy to assess early AMD. In clinical settings, some of these tests are not available, take a long time to administer, and/or are not standardized. Among the psychophysical tests of vision, logMAR visual acuity (VA) or Snellen charts are still the most commonly used primary outcome measure in clinical trials designed to address AMD.

Evidence exists to indicate that tests of photopic lowcontrast visual acuity (LC-VA) are predictors of significant subsequent VA loss in elderly subjects with good initial visual acuity. ${ }^{16,17}$ Although some studies have suggested that patients with AMD show a reduction in photopic LC-VA, reports on the usefulness of photopic LC-VA for diagnosing early AMD have been conflicting. ${ }^{18-20}$ Few studies have centered on assessing mesopic VA in AMD. In one such study, significantly reduced near-letter VA was detected using the Smith-Kettlewell Low Luminance (SKILL) card in subjects with early AMD, ${ }^{21}$ and a relatively greater loss of distance HC-VA was detected under low luminance conditions in eyes with GA. ${ }^{4}$ Further, the extent of distance VA loss in low luminance conditions at baseline for GA subjects was predictive of subsequent VA loss at 2 years. ${ }^{22}$ However, as far as we know, no study has been designed to compare mesopic distance VA in eyes with early AMD and control eyes, both with good photopic VA.

This study was designed to determine, using a standard logMAR chart, whether eyes with early AMD showed impaired 
mesopic distance HC-VA and LC-VA compared to healthy eyes that similarly had $20 / 25$ vision or better.

\section{METHODS}

\section{Subjects}

The study was conducted at the School of Optometry, Complutense University, Madrid, Spain. Subjects for the early AMD group were recruited from the Hospital del Henares, Madrid. Control subjects were relatives of those visiting the hospital or were recruited through advertisements placed at and around the university. Fifty subjects were enrolled, and measurements were obtained in 22 subjects with early AMD (age range 50-77 years; mean age 64.9 \pm 7 years) and 28 healthy control subjects (age range 51-78; mean age $64.6 \pm 7$ years). Mean age was not significantly different between the two groups. Only the right eye of each subject was measured.

All eyes underwent a careful ophthalmologic examination, including visual acuity, subjective refraction, slit-lamp biomicroscopy of the anterior segment, and dilated fundoscopy and digital color photographs with a 3D OCT-1000 (Topcon Corporation, Tokyo, Japan). Subjects were required to be older than 50 years of age, to have a bestcorrected visual acuity of 0.8 decimal (20/25 Snellen) or better in at least one eye, a refractive error not greater than \pm 3.75 diopters (D) sphere or \pm 1.50 D cylinder, and normal color vision. Exclusion criteria were posterior subcapsular cataract; cortical or nuclear opacities greater than LOCS III classification grade $2^{23}$; diabetic retinopathy; glaucoma; amblyopia; retinal vascular disease; or any other retinal abnormality. Subjects were also excluded if they were aphakic or pseudophakic or had undergone retinal surgery.

Early AMD was defined according to the International Classification and Grading System for $\mathrm{AMD}^{2}$ as the presence of soft drusen $(\geq 63 \mu \mathrm{m})$ with or without RPE abnormalities in the form of hyperpigmentation and/or hypopigmentation based on photographic grading and the templates of the Age-Related Eye Disease Study (AREDS) Group. ${ }^{24}$ Control subjects were required to show no visible signs of AMD in the tested eye (a few hard indistinct drusen $<63 \mu \mathrm{m}$ were permitted, no RPE pigmentary alterations).

The guidelines of the Declaration of Helsinki were adhered to, and full approval for the study was obtained from our institution's review board. Subjects were informed about the study protocol before giving their consent to participate.

\section{Visual Acuity}

Best-corrected logMAR VA was measured monocularly with best spectacle correction using high-contrast (96\%) and low-contrast (10\%) Bailey-Lovie logMAR letter charts under both photopic $\left(85 \mathrm{~cd} / \mathrm{m}^{2}\right)$ and mesopic $\left(0.1-0.2 \mathrm{~cd} / \mathrm{m}^{2}\right)$ luminance conditions at a distance of 4 meters. Subjects were forced to guess letters even if they were unsure. Each letter read correctly on each line was given a score of $0.02 \mathrm{log}$ units. In this chart, a loss of one line of letters corresponds to a logMAR increase of 0.1. The Bailey-Lovie chart is a valid, reliable, and rapid method of measuring threshold visual acuity for research purposes, ${ }^{25}$ and HC-VA and LC-VA acuity have been shown to be repeatable. In previous studies the repeatability coefficient $(1.96 \mathrm{SD})$ was 0.07 to 0.1 logMAR or one line for the HC (90\%) chart. ${ }^{26-28}$ The limits of agreement increased to approximately $\pm 0.15 \log$ MAR for the LC (10\%) chart. ${ }^{26,29}$ Under dim illumination conditions, Applegate et al. recommended mesopic LC-VA as a sensitive clinically viable measure of visual performance in healthy eyes. ${ }^{30}$

The chart was externally illuminated with a halogen lamp behind a screen connected to a potentiometer to adjust the exact voltage needed to reach the adequate mesopic luminance level with the room lighting turned off. This setup provides uniform luminance over the chart. For photopic conditions, normal room lighting was left on. In both cases, measures of luminance for the tests were obtained using a
TABLE 1. Distance High-Contrast (HC) and Low-Contrast (LC) Visual Acuity Means (logMAR VA) Recorded in Photopic and Mesopic Luminance Conditions in the Early AMD and Control Groups

\begin{tabular}{lcclcc}
\hline & \multicolumn{2}{c}{ Photopic } & logMAR VA & & \multicolumn{2}{c}{ Mesopic logMAR VA } \\
\cline { 2 - 3 } \cline { 5 - 6 } Group & HC & LC & & HC & LC \\
\hline Early AMD & $0.03 \pm 0.07$ & $0.24 \pm 0.12$ & & $0.55 \pm 0.12$ & $1.00 \pm 0.11$ \\
Control & $0.06 \pm 0.05$ & $0.26 \pm 0.11$ & & $0.44 \pm 0.11$ & $0.72 \pm 0.13$ \\
\hline
\end{tabular}

MAVO-SPOT ${ }^{2}$ USB luminance meter (Gossen Lighting Control, Nuremberg, Germany). The subject was first tested under mesopic conditions; at least 10 minutes was allowed to dark adapt before the test. After this procedure, acuity testing was continued at photopic luminance levels.

\section{Statistical Analysis}

Visual acuity measurements were analyzed by mixed model ANOVA with one between-subjects factor (group [early AMD versus control]) and two within-subjects factors (luminance condition [mesopic versus photopic] and contrast [low versus high]). When interaction effects were significant, the data were then split by each factor, and Student's $t$-test used to test the difference between the means. A $P$ value less than 0.05 was taken to denote statistical significance. Statistical analysis was performed using SPSS Statistics 19 for Windows (SPSS Inc., IBM, Somers, NY).

The diagnostic value of each test was assessed by the area under the curve (AUC) of a Receiver Operating Characteristic (ROC) analysis as a plot of the sensitivity for AMD visual function abnormality against the false alarm rate $(1-$ specificity). We defined a test as valid when the AUC was $>0.70 .31$ The $\chi^{2}$ test was used to compare the AUCs. These analyses were performed using SigmaPlot 11 software (Systat Software, Inc., Chicago, IL)

\section{RESUlts}

Table 1 provides the mean values of photopic best-corrected HC-VA and LC-VA (logMAR) and mean mesopic HC-VA and LCVA (logMAR) recorded in the early AMD and control groups. Mixed ANOVA showed a main effect of the presence of early AMD $(F=12.95 ; P=0.001)$ and main effects of contrast $(F=$ 1659.24; $P<0.0001)$ and luminance condition $(F=1045.35 ; P$ $<0.0001)$. Significant interactions were detected between contrast and group $(F=42.79 ; P<0.0001)$; luminance condition and group $(F=46.73 ; P<0.0001)$; luminance condition and contrast $(F=102.65 ; P<0.0001)$; and group, contrast, and luminance condition $(F=28.01 ; P<0.0001)$. Interaction effects were examined using Student's $t$-test. Under photopic conditions, means for HC-VA and LC-VA did not vary significantly between the early AMD and control groups. In contrast, under mesopic conditions, the AMD group showed worse VA at both high and low contrast. Mean mesopic HC-VA was $0.1 \log$ MAR (one line of letters on the chart) worse in the AMD group than in the control group $(P=0.002)$, while mean mesopic LC-VA was 0.28 logMAR (nearly three lines of VA) worse $(P<0.0001)$ in the AMD group than the control group.

Figure 1 shows the mean differences in logMAR units recorded between LC-VA and HC-VA measured at both luminance levels in the two groups of eyes. While the mean differences in VA were similar for the two groups under photopic conditions $(P=0.82)$, our mesopic measurements revealed a difference between mean LC-VA and HC-VA of 0.45 \pm 0.06 logMAR ( 4.5 lines of VA) for the AMD group and of 0.27 \pm 0.06 logMAR (2.7 lines) for the control group, that is, nearly two lines of difference $(0.18 \log$ MAR $)$ between the mesopic means of the two groups $(P<0.0001)$. 


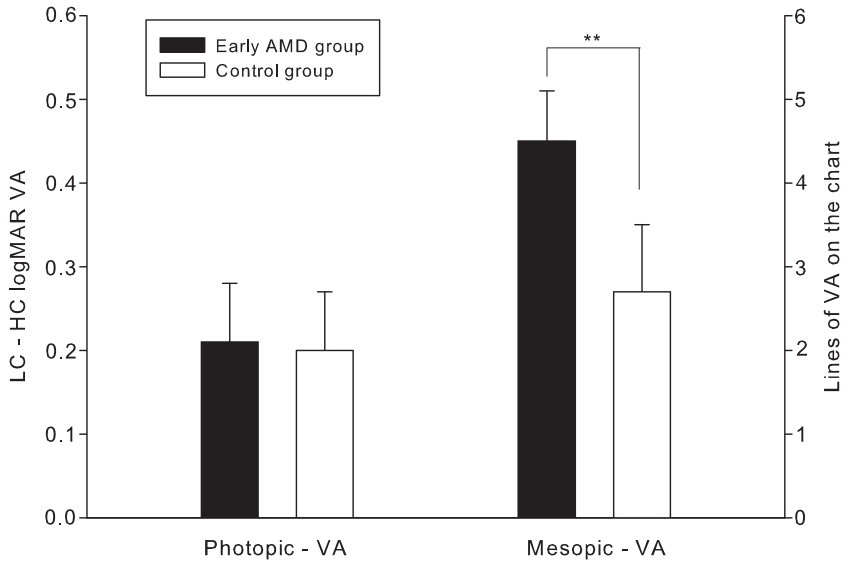

Figure 1. Mean difference between the HC-VA and the LC-VA measurements made under photopic or mesopic luminance conditions in the early AMD and control groups. Mean VA differences are plotted as logarithms of the minimum angle of resolution (logMAR units) on the left y-axis and as numbers of lines on the chart on the right y-axis. **P $P<0.01$

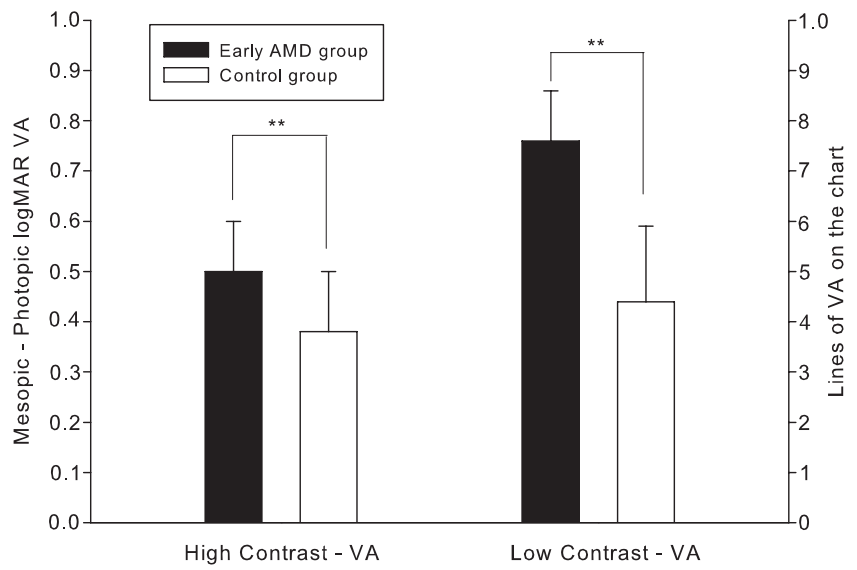

Figure 2. Mean difference between the mesopic and photopic VA measurements made using both high- and low-contrast letter charts in the early AMD and control groups. Mean visual acuity differences are plotted as logarithms of the minimum angle of resolution (logMAR units) on the left y-axis and as numbers of lines on the chart on the right y-axis. ${ }^{* *} P<0.01$.

Figure 2 shows the mean differences in logMAR units detected between mesopic and photopic VA in both high- and low-contrast tests in the AMD and control groups. The mean drop in HC-VA observed under mesopic compared to photopic conditions was 5.1 lines (a 0.51 logMAR increase from 0.55 [mesopic] to 0.03 [photopic]) in the AMD group and 3.8 lines (0.38 logMAR) in the control group, that is, 1.3 lines $(0.13$ logMAR) of difference between the means of the two groups ( $P$ $<0.0001)$. For LC-VA, the mean difference observed when photopic with mesopic conditions were compared was 7.6 lines $(0.76 \log M A R)$ in the early AMD group and 4.4 lines $(0.44$ $\operatorname{logMAR})$ in the control group, that is, 3.2 lines of difference (0.32 logMAR) between the means of the two groups $(P<$ $0.0001)$.

In the ROC analyses (Table 2), the AUC for photopic HC-VA and LC-VA was below the 0.70 value, indicating no diagnostic capacity. Mesopic LC-VA yielded the highest AUC value $(0.94 \pm$ 0.030 ), indicating a lower false alarm rate, the difference with the mesopic HC-VA AUC $(0.76 \pm 0.067)$ being statistically significant $(P=0.0001)$. Comparable AUC values were found between mesopic LC-VA $(0.94 \pm 0.030)$ versus VA difference (mesopic LC-VA - photopic LC-VA) $(0.96 \pm 0.028)(P=0.389)$ and mesopic LC-VA $(0.94 \pm 0.030)$ versus VA difference (mesopic LC-VA - mesopic HC-VA) $(0.96 \pm 0.027)(P=0.625)$. Table 3 shows sensitivity and specificity values for ROC curves with AUC values higher than 0.70. These measurements emerged as sensitive and specific for detecting early AMDrelated functional abnormality.

\section{Discussion}

The findings of our study indicate impaired mesopic bestcorrected distance HC-VA and LC-VA in eyes with early AMD compared to healthy control eyes. Under photopic conditions, both mean HC-VA and LC-VA did not vary significantly between the early AMD and control groups. It should be noted that all eyes had a photopic distance HC-VA of $20 / 25$ or better so that confounding effects could be avoided. Other studies have shown reductions of a few letters in distance photopic LC-VA in AMD patients, ${ }^{18}$ and Lovie-Kitchin ${ }^{19}$ and Abadi and Pantazidou $^{20}$ concluded that while photopic LC-VA was reduced in AMD, its measurement gave no additional information over that provided by HC-VA. In these studies, the AMD eyes already had slightly reduced HC-VA; thus this conclusion is logical.

While photopic HC-VA measurement is inadequate for the assessment of functional deficits in early $\mathrm{AMD}^{17}$ or for monitoring progression of the disorder, ${ }^{32}$ according to our results, VA measures under mesopic luminance conditions could be useful. We found that mean distance HC-VA and LC-VA were one line and 2.8 lines of letters worse, respectively, in the early AMD group than in the control group (Table 1). Also, in mesopic conditions the difference between LC-VA and HC-VA was significantly greater in the early AMD group-1.8 lines on average more than in the control group (Fig. 1). Only a few studies have examined VA under low luminance conditions in early AMD subjects. Feigl et al. recorded significantly reduced SKILL scores (difference in number of letters between the dark and light sides of the card) using the SKILL card ${ }^{33}$ in people with early AMD (and an HC-VA of 6/12 or better). ${ }^{21}$ It should be noted that the test was conducted at near distance under normal room lighting conditions and that the early AMD subjects examined already had slightly reduced HC-VA. In eyes with GA, a relatively greater loss of distance HC-VA (4.6 lines) was detected when compared to eyes with drusen (2.2 lines), that is, 2.4 lines of difference, under low luminance conditions obtained using a neutral density filter. ${ }^{4}$ Our mesopic condition was one of very low luminance, and the mean HC-VA drop

TABLE 2. ROC Analyses of Photopic and Mesopic High-Contrast (HC) and Low-Contrast (LC) VA and for Differences between Them

\begin{tabular}{lllr}
\hline VA Parameters & AUC \pm SD & VA Difference Parameters & AUC \pm SD \\
\hline Photopic HC-VA & $0.67 \pm 0.084$ & Photopic LC-VA - photopic HC-VA & $0.50 \pm 0.087$ \\
Photopic LC-VA & $0.63 \pm 0.083$ & Mesopic HC-VA - photopic HC-VA & $0.81 \pm 0.060$ \\
Mesopic HC-VA & $0.76 \pm 0.067$ & Mesopic LC-VA - photopic LC-VA & $0.96 \pm 0.028$ \\
Mesopic LC-VA & $0.94 \pm 0.030$ & Mesopic LC-VA - mesopic HC-VA & $0.96 \pm 0.027$ \\
\hline
\end{tabular}


TABLE 3. Sensitivity and Specificity Values for Each ROC Curve (AUC $>0.70$ ) of Photopic and Mesopic High-Contrast $(\mathrm{HC})$ and Low-Contrast (LC) VA for Detecting Early AMD-Related Functional Abnormality

\begin{tabular}{|c|c|c|c|c|}
\hline Cutoff & Sensitivity, \% & $95 \% \mathrm{CI}$ & Specificity, \% & $95 \% \mathrm{CI}$ \\
\hline \multicolumn{5}{|c|}{ Mesopic HC-VA } \\
\hline 0.45 & 68.2 & $(45.1-86.1)$ & 64.9 & $(44.1-81.4)$ \\
\hline 0.51 & 59.1 & $(36.4-79.3)$ & 82.1 & $(63.1-93.9)$ \\
\hline 0.55 & 40.9 & $(20.7-63.7)$ & 82.1 & $(63.1-93.9)$ \\
\hline 0.59 & 31.8 & $(13.9-54.9)$ & 85.7 & $(67.3-96.0)$ \\
\hline 0.65 & 27.3 & $(10.7-50.2)$ & 96.4 & $(81.7-99.9)$ \\
\hline \multicolumn{5}{|c|}{ Mesopic LC-VA } \\
\hline 0.81 & 100 & $(84.6-100)$ & 82.1 & $(63.1-93.9)$ \\
\hline 0.85 & 90.9 & $(70.8-98.9)$ & 85.7 & $(67.3-96.0)$ \\
\hline 0.89 & 77.3 & $(54.6-92.9)$ & 85.7 & $(67.3-96.0)$ \\
\hline 0.95 & 63.6 & $(40.7-82.8)$ & 92.9 & $(76.5-99.1)$ \\
\hline 1.01 & 36.4 & $(17.2-59.3)$ & 100 & $(87.7-100)$ \\
\hline \multicolumn{5}{|c|}{ Mesopic HC-VA - photopic HC-VA } \\
\hline 0.42 & 86.4 & $(65.1-97.1)$ & 67.7 & $(47.7-84.0)$ \\
\hline 0.49 & 63.7 & $(40.7-82.8)$ & 78.6 & $(59.1-91.7)$ \\
\hline 0.51 & 40.9 & $(20.7-63.7)$ & 82.1 & $(63.1-93.9)$ \\
\hline 0.55 & 31.8 & $(13.9-54.9)$ & 89.3 & $(71.8-97.7)$ \\
\hline 0.61 & 18.2 & $(5.2-40.3)$ & 96.4 & $(81.7-99.9)$ \\
\hline \multicolumn{5}{|c|}{ Mesopic LC-VA - photopic LC-VA } \\
\hline 0.61 & 95.5 & $(77.7-99.9)$ & 85.2 & $(66.3-95.8)$ \\
\hline 0.65 & 86.4 & $(65.1-97.1)$ & 92.6 & $(75.7-99.1)$ \\
\hline 0.71 & 59.1 & $(36.4-79.3)$ & 96.3 & $(81.0-99.9)$ \\
\hline 0.75 & 45.5 & $(24.4-67.8)$ & 96.3 & $(81.0-99.9)$ \\
\hline 0.85 & 27.3 & $(10.7-50.2)$ & 100 & $(87.2-100)$ \\
\hline \multicolumn{5}{|c|}{ Mesopic LC-VA - mesopic HC-VA } \\
\hline 0.35 & 95.5 & $(77.2-99.9)$ & 85.7 & $(67.3-96.0)$ \\
\hline 0.39 & 86.4 & $(65.1-97.1)$ & 92.9 & $(76.5-99.1)$ \\
\hline 0.41 & 77.3 & $(54.6-92.2)$ & 96.4 & $(81.7-99.9)$ \\
\hline 0.43 & 68.2 & $(45.1-86.1)$ & 96.4 & $(81.7-99.9)$ \\
\hline 0.47 & 27.3 & $(10.7-50.2)$ & 100 & $(87.7-100)$ \\
\hline
\end{tabular}

CI: confidence interval.

observed was 5.1 lines for eyes with early AMD versus 3.8 lines for control eyes, that is, 1.3 lines of difference between the means of the two groups. The mean LC-VA drop (between mesopic and photopic) was 3.2 lines more for the early AMD group compared to the control group (Fig. 2). These results indicate adversely affected VA under mesopic conditions in eyes with early AMD. It should be noted that in our study, measuring VA under low luminance conditions showed a good capacity to quantify functional abnormalities in eyes affected by early AMD; AUC values in the ROC analyses ranged from $0.76 \pm 0.067$ to $0.96 \pm 0.027$ (Table 2) and showed a significantly greater diagnostic capacity than the universally used measure of photopic HC-VA. These significant functional abnormalities were present despite the majority of participants having a photopic HC-VA of 20/25 or better.

Psychophysical studies have demonstrated that rod- and cone-mediated mesopic visual function deteriorates in the early stages of AMD. ${ }^{6}$ Mesopic VA is probably a better reflection of the role played by cone foveal function than by rod function under reduced illumination given the paucity of rods in the foveal region. It is still not clear whether functional deficits in early AMD measured using various psychophysical tests are primarily caused by a reduced sensitivity of photoreceptors, by postreceptor damage, or by damage to other tissues involved in AMD such as the RPE/Bruch's membrane complex or the choroid. Feigl et al. ${ }^{34}$ reviewed this issue, and based on their psychophysical and electrophysiological findings proposed that most function impairment in early AMD starts postreceptorally. For the purpose of our study, the so-called ischemia postreceptoral hypothesis could explain why mesopic VA is reduced in early AMD when the cones and photopic VA are still preserved.

The mesopic distance VA impairment detected in our study indicates that VA assessment using logMAR charts in low luminance conditions is a sensitive indicator of impaired macular function in the early stages of AMD before any photopic HC-VA alterations occur. This simple, inexpensive, and rapid measure of visual function could be especially useful for the early detection of AMD.

\section{References}

1. Friedman DS, O'Colmain BJ, Munoz B, et al. Prevalence of agerelated macular degeneration in the United States. Arch Ophthalmol. 2004;122:564-572.

2. Bird AC, Bressler NM, Bressler SB, et al. An international classification and grading system for age-related maculopathy and age-related macular degeneration. The International ARM Epidemiological Study Group. Surv Ophthalmol. 1995;39: 367-374

3. Brown B, Kitchin JL. Dark adaptation and the acuity/ luminance response in senile macular degeneration (SMD). Am J Optom Physiol Opt. 1983;60:645-650.

4. Sunness JS, Rubin GS, Applegate CA, et al. Visual function abnormalities and prognosis in eyes with age-related geographic atrophy of the macula and good visual acuity. Ophthalmology. 1997;104:1677-1691.

5. Scilley K, Jackson GR, Cideciyan AV, Maguire MG, Jacobson SG, Owsley C. Early age-related maculopathy and self-reported visual difficulty in daily life. Ophthalmology. 2002;109:12351242.

6. Neelam K, Nolan J, Chakravarthy U, Beatty S. Psychophysical function in age-related maculopathy. Surv Ophthalmol. 2009; 54:167-210.

7. Klein R, Wang Q, Klein BE, Moss SE, Meuer SM. The relationship of age-related maculopathy, cataract, and glaucoma to visual acuity. Invest Ophthalmol Vis Sci. 1995;36:182191.

8. Petzold A, Plant GT. Clinical disorders affecting mesopic vision. Ophthalmic Physiol Opt. 2006;26:326-341.

9. Curcio CA, Owsley C, Jackson GR. Spare the rods, save the cones in aging and age-related maculopathy. Invest Ophthalmol Vis Sci. 2000;41:2015-2018.

10. Feigl B, Brown B, Lovie-Kitchin J, Swann P. The rod-mediated multifocal electroretinogram in aging and in early age-related maculopathy. Curr Eye Res. 2006;31:635-644.

11. Owsley C, Jackson GR, White M, Feist R, Edwards D. Delays in rod-mediated dark adaptation in early age-related maculopathy. Ophthalmology. 2001;108:1196-1202.

12. Scholl HP, Bellmann C, Dandekar SS, Bird AC, Fitzke FW. Photopic and scotopic fine matrix mapping of retinal areas of increased fundus autofluorescence in patients with age-related maculopathy. Invest Ophthalmol Vis Sci. 2004;45:574-583.

13. Feigl B, Cao D, Morris CP, Zele AJ. Persons with age-related maculopathy risk genotypes and clinically normal eyes have reduced mesopic vision. Invest Ophthalmol Vis Sci. 2011;52: 1145-1150.

14. Lovie-Kitchin J, Feigl B. Assessment of age-related maculopathy using subjective vision tests. Clin Exp Optom. 2005;88:292303.

15. Hogg RE, Chakravarthy U. Visual function and dysfunction in early and late age-related maculopathy. Prog Retin Eye Res. 2006;25:249-276 
16. Schneck ME, Haegerstrom-Portnoy G, Lott LA, Brabyn JA, Gildengorin G. Low contrast vision function predicts subsequent acuity loss in an aged population: the SKI study. Vision Res. 2004;44:2317-2325.

17. Haegerstrom-Portnoy G. The Glenn A. Fry Award Lecture 2003: Vision in elders-summary of findings of the SKI study. Optom Vis Sci. 2005;82:87-93.

18. Kleiner RC, Enger C, Alexander MF, Fine SL. Contrast sensitivity in age-related macular degeneration. Arch Ophthalmol. 1988;106:55-57.

19. Lovie-Kitchin JE. High contrast and low contrast visual acuity in age related macular degeneration. Clin Exp Optom. 1989; 72:79-83.

20. Abadi RV, Pantazidou M. Low contrast letter acuity in agerelated maculopathy. Ophthalmic Physiol Opt. 1996;16:455459.

21. Feigl B, Brown B, Lovie-Kitchin J, Swann P. Cone-mediated multifocal electroretinogram in early age-related maculopathy and its relationships with subjective macular function tests. Curr Eye Res. 2004;29:327-336.

22. Sunness JS, Rubin GS, Broman A, Applegate CA, Bressler NM, Hawkins BS. Low luminance visual dysfunction as a predictor of subsequent visual acuity loss from geographic atrophy in age-related macular degeneration. Ophthalmology. 2008;115: 1480-1488.

23. Chylack LT Jr, Wolfe JK, Singer DM, et al. The Lens Opacities Classification System III. The Longitudinal Study of Cataract Study Group. Arch Ophthalmol. 1993;111:831-836.

24. Age-Related Eye Disease Study Research Group. The AgeRelated Eye Disease Study system for classifying age-related macular degeneration from stereoscopic color fundus photographs: the Age-Related Eye Disease Study Report Number 6. Am J Ophthalmol. 2001;132:668-681.
25. Lovie-Kitchin J. Visual acuity measures in clinical research. Ophthalmic Physiol Opt. 1989;9:456.

26. Cho P, Woo GC. Repeatability of the Waterloo four-contrast LogMAR visual acuity chart and near vision test card on a group of normal young adults. Ophthalmic Physiol Opt. 2004; 24:427-435.

27. Leinonen J, Laakkonen E, Laatikainen L. Random measurement error in visual acuity measurement in clinical settings. Acta Ophthalmol Scand. 2005;83:328-332.

28. Arditi A, Cagenello R. On the statistical reliability of letterchart visual acuity measurements. Invest Ophthalmol Vis Sci. 1993;34:120-129.

29. Hazel CA, Elliott DB. The dependency of LogMAR visual acuity measurements on chart design and scoring rule. Optom Vis Sci. 2002;79:788-792.

30. Applegate RA, Marsack JD, Thibos LN. Metrics of retinal image quality predict visual performance in eyes with 20/17 or better visual acuity. Optom Vis Sci. 2006;83:635-640.

31. Linden A. Measuring diagnostic and predictive accuracy in disease management: an introduction to receiver operating characteristic (ROC) analysis. J Eval Clin Pract. 2006;12:132139.

32. Sarks JP, Sarks SH, Killingsworth MC. Evolution of geographic atrophy of the retinal pigment epithelium. Eye (Lond). 1988; 2(Pt 5):552-577.

33. Haegerstrom-Portnoy G, Brabyn J, Schneck ME, Jampolsky A. The SKILL Card. An acuity test of reduced luminance and contrast. Smith-Kettlewell Institute Low Luminance. Invest Ophthalmol Vis Sci. 1997;38:207-218.

34. Feigl B, Brown B, Lovie-Kitchin J, Swann P. Functional loss in early age-related maculopathy: the ischaemia postreceptoral hypothesis. Eye (Lond). 2007;21:689-696. 